\title{
Trends of transfusion transmittable infections among voluntary blood donors in a cardiac care hospital, Bengaluru, India
}

Shivalingegowda S. ${ }^{1}$, Banandur Nanjundappa K. ${ }^{2 *}$, Kannan U. ${ }^{3}$, Cholenahalli Nanjappa M. ${ }^{4}$ DOI: https://doi.org/10.17511/ijmrr.2019.i02.14

1 Sathyavathi Shivalingegowda, Assistant Professor, Department of Pathology and Blood Bank, Sri Jayadeva Institute of Cardiovascular Sciences and Research, Bengaluru, Karnataka, India.

2* Krishnamurthy Banandur Nanjundappa, Assistant Professor, Department of Pathology and Blood Bank, Sri Jayadeva Institute of Cardiovascular Sciences and Research, Bengaluru, Karnataka, India.

3 Umadevi Kannan, Associate Professor and HOD, Department of Blood Bank, Sri Jayadeva Institute of Cardiovascular Sciences and Research, Bengaluru, Karnataka, India.

4 Manjunath Cholenahalli Nanjappa, Director, Professor and HOD, Department of Cardiology, Sri Jayadeva Institute of Cardiovascular Sciences and Research, Bengaluru, Karnataka, India.

Introduction: Transmission of infectious diseases through donated blood is of concern to blood safety as transfusion forms an integral part of medical and surgical therapy. Blood transfusion carries the risk of transfusion-transmittable infections including HIV, hepatitis-B etc. Screening of voluntary donors who represent healthy population serves as a predictor for these dreadful diseases in healthy population. Materials and Methods: This retrospective study was conducted at the blood bank of Sri Jayadeva Institute of Cardiovascular Sciences and Research, Bengaluru. Data were analyzed for a period of 7 years from January 2011 to December 2017. All voluntary donors including replacement donors of our blood bank were screened for Hepatitis B Virus (HBV), Hepatitis $C$ Virus (HCV) and Human Immunodeficiency Virus (HIV) and Syphilis infection by using ELISA. Results: The most common infection was Hepatitis B (0.69\%) followed by Syphilis $(0.34 \%)$ and HIV $(0.0736 \%)$ and least with HCV $(0.04 \%)$ in our study. Conclusion: This study has shown a decrease in seroprevalence for HIV and increase in seroprevalence for HCV over 7 years study period.

Keywords: Voluntary donors, Replacement donors, HIV, HBV, HCV, HBsAg, VDRL

\section{Corresponding Author}

Krishnamurthy Banandur Nanjundappa, Assistant Professor, Department of Pathology and Blood Bank, Sri Jayadeva Institute of Cardiovascular Sciences and Research, Bengaluru, Karnataka, India.

Email: drbanandur@yahoo.co.in
How to Cite this Article

Shivalingegowda S, Nanjundappa KB, Kannan U, Nanjappa MC. Trends of transfusion transmittable infections among voluntary blood donors in a cardiac care hospital, Bengaluru, India. Int J Med Res Rev. 2019;7(2):139-142.

Available From

https://ijmrr.medresearch.in/index.php/ijmrr/article/

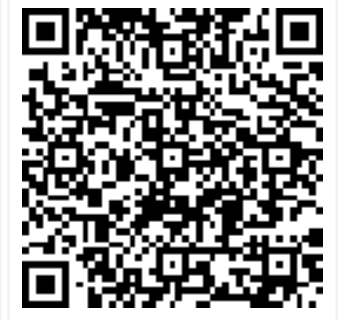
view/1047

Review Round 1
2019-01-14
Funding
Nil

Note 


\section{Introduction}

India, the second most populous nation in the world is classified as an intermediate HBV endemic (HBsAg carriage 2-7\%) zone and has the second largest global pool of chronic HBV infections [1]. $\mathrm{HCV}$ is a leading cause of chronic liver diseases, viz., hepatic fibrosis, cirrhosis, end-stage liver disease and hepatocellular carcinoma (HCC) and in India alone there are about 12-13 million HCV carriers and modeling data predict that the burden of disease could soon increase substantially [2].

Transmission of infectious diseases through donated blood is of concern to blood safety as transfusion forms an integral part of medical and surgical therapy. Blood transfusion carries the risk of transfusion-transmittable infections, including HIV, Hepatitis-B, syphilis, malaria and infrequently toxoplasmosis. With every unit of blood, there is $1 \%$ chance of transfusion-associated problems including transfusion-transmitted diseases [3]. Among all infections, HIV and Hepatitis-B are the most dreadful. The first case of transfusion-associated AIDS was described in an infant given transfusion for Erythroblastosis foetalis [4].

According to the National AIDS Control Organization (NACO) guidelines all blood sample must be tested for Human Immunodeficiency Virus (HIV) 1 and 2, Hepatitis B, Hepatitis C, syphilis and malaria [5]. In spite of all these guidelines we still have cases of transfusion transmitted infections.

Voluntary donors are screened as they represent healthy population at large. So indirectly we will be able to know the seroprevalence of these diseases in healthy population.

This study was taken up to know the trends of HIV, Hepatitis-B, Hepatitis C and syphilis infections among donors over a period of 7 years in Bengaluru.

\section{Material and Methods}

The objective of this study is to estimate the seroprevalence of HIV, HBV, HCV, VDRL and their seroprevalence trends over 7 years among voluntary blood donors at a cardiac care hospital in Bengaluru. This retrospective study was conducted at the blood bank of Sri Jayadeva Institute of Cardiovascular sciences \&Research, Bengaluru. Data was collected for a period of 7 years from January 2011 to December 2017.
Tests were routinely done on every blood unit collected to exclude HIV, HBV, HCV, Syphilis and Malaria. Donors were carefully selected for donation by trained personnel after a complete physical examination and satisfactorily answering the donor's questionnaire. The family members, friends or relatives of the patients were categorized as replacement donors. People who donate blood without expecting any favor in return or in voluntary blood donation camps were categorized as voluntary blood donors. The eligibility criteria for the donors, i.e. age between 18 and 60 years, minimum weight $45 \mathrm{~kg}$, hemoglobin $>12 \mathrm{gms} / \mathrm{dl}$, with no history of HIV, HBV \& HCV or any other sexually transmitted infections, no history of jaundice in past 1 year was strictly adhered to for recruitment of blood donors. Care was taken to maintain confidentiality of data while conducting the study.

The screening for HIV was done by 4th generation ELISA using kits from Bio-Rad Genscreen Year ULTRA HIV Ag- Ab. HBsAg was detected by Bio-Rad MONOLISHA HBsAg ULTRA. Anti HCV test was done by ELISA - Bio-Rad MONOLISHA Anti HCV PLUS Version 3. Syphilis was screened by Bio-Rad Syphilis total Ab. Manufacturer's instructions were followed.

Quality Control: Internal quality controls were performed daily by using both positive and negative controls from the manufacturers.

\section{Results}

A total of 44,778 blood donors were for screened for HIV, HBV, HCV and Syphilis. Table 1 shows seroprevalence of transfusion transmittable infections. The overall highest prevalence was seen for HBsAg $(0.69 \%)$ followed by syphilis $(0.34 \%)$, HIV $(0.0736 \%)$, and HCV $(0.04 \%)$. There is a decreasing percentage of HIV from 2011 to 2017 that ranges from $0.05 \%$ to 0.016 . Varying trends in seroprevalence of HBV has been seen \& it ranges between $0.47 \%$ to $0.78 \%$. There varying trends of HCV has been noted over 7 years that is $0.00016 \%$ to $0.07 \%$. The seroprevalence of syphilis also shows variable trend that is from $0.24 \%$ to $0.52 \%$.

Table-1: Seroprevalence of transfusion transmittable infections among donor blood samples.

\begin{tabular}{|l|l|c|c|c|c|}
\hline \multicolumn{1}{|c|}{$\begin{array}{c}\text { Total no. of } \\
\text { Donors }\end{array}$} & $\begin{array}{c}\text { HIV } \\
(\%)\end{array}$ & $\begin{array}{c}\text { HBsAg } \\
(\%)\end{array}$ & $\begin{array}{c}\text { HCV } \\
(\%)\end{array}$ & $\begin{array}{c}\text { SYPHILIS } \\
(\%)\end{array}$ \\
\hline 2011 & 5,892 & $3(0.05)$ & $43(0.72)$ & $2(0.03)$ & $17(0.28)$ \\
\hline 2012 & 6,648 & $4(0.06)$ & $52(0.78)$ & $4(0.06)$ & $21(0.31)$ \\
\hline 2013 & 7,029 & $8(0.11)$ & $55(0.78)$ & $3(0.04)$ & $30(0.42)$ \\
\hline
\end{tabular}




\begin{tabular}{|l|l|l|l|l|l|}
\hline 2014 & 6,662 & $5(0.07)$ & $51(0.76)$ & $3(0.04)$ & $18(0.27)$ \\
\hline 2015 & 6,541 & $3(0.04)$ & $49(0.74)$ & $5(0.07)$ & $16(0.24)$ \\
\hline 2016 & 5,918 & $3(0.05)$ & $33(0.55)$ & $1(0.01)$ & $31(0.52)$ \\
\hline 2017 & 6,088 & $7(0.016)$ & $29(0.476)$ & $1(0.00016)$ & $20(0.328)$ \\
\hline Total & 44,778 & $33(0.0736)$ & $312(0.69)$ & $19(0.04)$ & $153(0.34)$ \\
\hline
\end{tabular}

\section{Discussion}

Blood transfusion is an integral and life-saving procedure of modern medicine, but simultaneously it carries the risk of transmitting lifethreatening transfusion transmittable infections. Blood transfusion is a life-saving intervention that has an essential role in patient management within health care systems. WHO recommends following an integrated strategy for the provision of safe blood and blood products and safe, efficacious blood transfusion and recommends a highly sensitive and specific screening test using Immunoassay methods to screen for HIV, HBV, HCV and Syphilis. Wellorganized blood banks that can provide safe blood should be established nationwide to provide for the transfusion needs of the patient population [6]. As there is a huge demand of blood and its components by patients for transfusion therapy, even a low viral load blood unit may cause infection in the recipient. Therefore, it is very important that the blood banks have effective screening systems to detect, segregate and remove reactive blood and its components. Only non-reactive blood and blood components should be released for the safe transfusion to the patients. In 2002, "An action plan for blood safety" a National Blood Policy was adopted by the Government of India to ensure safe blood supply in the country as standards of blood banks within the country varied from state to state. The policy was formulated to ensure easy and adequate supply of safe blood and blood components to the patients required which is free from transfusion transmitted infections, and is stored and transported under optimum conditions and also to transfuse the blood and its components under supervision of trained personnel for all who need it irrespective of their economic or social status.

Transmission of $\mathrm{HCV}$ is primarily through blood exposure, and majority progress to chronic infection with increasing chances of cirrhosis and hepatocellular carcinoma compared to that of HBV. In our study, HCV seroprevalence was $0.04 \%$, which is more than in different Indian studies where $\mathrm{HCV}$ seroprevalence ranged between 0.00016 to $0.07 \%$ [7-11].
But we observed there is varying trends in the prevalence of HCV over the 7 years study period, highest prevalence of about $0.07 \%$ was noted in 2015 and low prevalence of $0.00016 \%$ in 2017 . This might be because of unawareness about its mode of transmission and chronicity. Meena et al [12]. revealed that the prevalence of $\mathrm{HCV}$ infection among blood donors showed a significant increasing trend from $0.18 \%$ in 2005 to $0.82 \%$ in 2009 . Their study was based on the sero-reactivity in anti $\mathrm{HCV}$ ELISA-based assay. This is significant because HCV is very infectious and also its way to chronicity. A healthy donor who is unaware of such infection is a definite threat to community in spreading the disease.

We observed a seroprevalence of $0.0736 \%$ for HIV. Gupta et al [10] and Tiwari et al [13] reported $0.084 \%$ and $0.054 \%$ prevalence of HIV among blood donors. We found a decrease in seroprevalence of HIV, from $0.11 \%$ in 2013 to $0.0 .016 \%$ in 2017 and this could be attributed for many reasons for it. Over the years, awareness of spread of HIV might have improved among the population, making voluntary blood donors with risk behavior abstain from donating blood. Also, better training and awareness of health workers at blood banks may be effective in screening out those with high risk of HIV. An increase in HIV incidence from 0.04 to $0.55 \%$ was shown in New Delhi between 1989 and 1995, whereas a decreasing trend of HIV incidence $(0.81 \%$ in $2006,0.32 \%$ in 2007 , and $0.53 \%$ in 2008 , overall $0.51 \%$ ) was noted in blood donors of Bhopal [9], [10]. Incidence of HIV (0.16$0.18 \%$ ) in Kerala remained constant from 1990 to 1999 [7]. In a study conducted by Chandra T et al [16] it was found that the prevalence of HIV had been decreasing in the Indian population similar to our study supporting the growing awareness of this life threatening diseases.

The seroprevalence of HBsAg was $0.69 \%$ in our study. Voluntary blood donors of Chandigarh had $0.66 \%$ seropositivity of HBV [11]. Even the rural population of Ambajogai, India, had very high prevalence of HBV (4.84\%) [14]. In study done by Chandra.et al [15]. (2001-2007), it was observed that the most common infection was hepatitis B $(1.96 \%)$ followed by $\mathrm{HCV}(0.85 \%)$ and HIV $(0.23 \%)$, and $0.01 \%$ in syphilis. HBV infection if it coinfects with HIV or HCV is a definitive risk factor.

Wenoted a few cases of co-infections in our study that a total of six cases of HIV with HBsAg and one case of HIV with Syphilis. 
In low endemic areas like the south east Asian countries and India transfusion transmission infections rate has reduced due to usage of better diagnostic and screening tests [1]. In a study conducted by Sawke et al, HbsAg had the highest prevalence rate compared to other transfusion transmittable infections, which was similar to our present study [10]. Transmission of Syphilis is not a threat due to the usage of good antibiotics but the VDRL reactivity points towards the highrisk behavior of the donor and further being at risk of developing other infections like HIV and HBV [10]. For syphilis, the seroprevalence was found to be $0.34 \%$ in our study, which was much lower than reported by other studies $0.85 \%$ and $1.2 \%$. HBsAg infection in spite of decreasing prevalence still continuous to be a greater threat to the general population due to its high incidence. As detection of HbsAg antibodies is not possible during window period, newer markers like HBc-IgM is shown to be a useful tool and is being suggested to be included as one of the screening methods for detection [10]. There has been a significant reduction in the prevalence of HIV and $\mathrm{HCV}$ mostly due to the availability of highly specific and sensitive diagnostic kits and also due to increased awareness among the blood donors. Knowing the seroprevalence of HIV, HBV, HCV and Syphilis among voluntary donors is valuable information which will help us to know the hidden percentage of infections that prevails in the community. If these voluntary blood donors found to have transfusion-transmissible infections need to be educated about the occurrence and the spread of these infections in the community.

\section{Conclusion}

The highest prevalence was seen for HBsAg $(0.69 \%)$ followed by Syphilis $(0.34 \%)$, then HIV $(0.0736 \%)$ and least with HCV $(0.04 \%)$ in our study. We found little variations in seroprevalence of transfusion-transmissible infections over the 7 years study period. Improvements must be made in donor selection criteria and screening for infectious diseases in order to provide a safe blood supply. Blood can save lives; however, it still carries the potential to transmit life-threatening infections.

\section{Reference}

01. Lavanchy D. Hepatitis B virus epidemiology, disease burden, treatment, and current and emerging prevention and control measures. J Viral Hepat. 2004 Mar;11(2)97-107. [Crossref]
02. Narahari S, Juwle A, Basak S, et al. Prevalence and geographic distribution of Hepatitis $C$ Virus genotypes in Indian patient cohort. Infect Genet Evol. 2009 Jul;9(4)643-5.

doi: 10.1016/j.meegid.2009.04.001 [Crossref]

03. Widmann FK, editor. Technical manual American association of blood banks. Aglington USA. $1985 ; 325-44$.

[Crossref]

04. Hollan SR, Wagstaff W, Leikola J, Lothe F. Management of blood transfusion services. Geneva- World Health Organization. 1990;131. [Crossref]

05. National AIDS Control Organization. Standards for Blood Banks and Blood Transfusion Services. New Delhi- Ministry of Health and Family Welfare Government of India. 2007. [Crossref]

06. WHO. Screening donated blood for transfusion. World Health Organization. 2010.

[Crossref]

07. Mathai J, Sulochana PV, Satyabhama S, et al. Profile of transfusion transmissible infections and associated risk factors among blood donors of Kerala. Indian J Pathol Microbiol. 2002 Jul;45(3)319-22.

[Crossref]

08. Srikrishna A, Sitalakshmi S, Damodar P. How safe are our safe donors?. Indian J Pathol Microbiol. 1999 Oct;42(4)411-6.

[Crossref]

09. Nanu A, Sharma SP, Chatterjee K, et al. Markers for transfusion-transmissible infections in north Indian voluntary and replacement blood donorsprevalence and trends 1989-1996. Vox Sang. $1997 ; 73(2) 70-3$.

[Crossref]

10. Sawke N, Sawke GK, Chawla S. Seroprevalence of Common Transfusion - Transmitted infections among Blood Donors. People's Journal of Scientific Research. 2010;3(1)5-8. [Crossref]

11. Gupta N, Kumar V, Kaur A. Seroprevalence of HIV, HBV, HCV and syphilis in voluntary blood donors. Indian J Med Sci. 2004 Jun;58(6)255-7. [Crossref] 
12. Meena M, Jindal T, Hazarika A. Prevalence of hepatitis $B$ virus and hepatitis $C$ virus among blood donors at a tertiary care hospital in Indiaa five-year study. Transfusion. 2011Jan;51(1)198-202.

doi: $10.1111 /$ j.1537-2995.2010.02801.x [Crossref]

13. Tiwari B, Ghimire $P$, Karkee S, Rajkarnikar M. Seroprevalence of human immunodeficiency Virus in Nepalese blood donors- A study from three regional blood transfusion services. Asian J Transf Sci. $2008 ; 2 ; 66-8$.

[Crossref]

14. Sonwane BR, Birare SD, Kulkarni PV. Prevalence of seroreactivity among blood donors in rural population. Indian J Med Sci. 2003 Sep;57(9)405-7.

[Crossref]
15. Chandra T, Kumar A, Gupta A. Prevalence of transfusion transmitted infections in blood donors- an Indian experience. Trop Doct. 2009 Jul;39(3)152-4.

doi: $10.1258 /$ td.2008.080330 [Crossref]

16. Chandra T, Rizvi SN, Agarwal D. Decreasing prevalence of transfusion transmitted infection in Indian scenario. Scientific World Journal. $2014 ; 173939$.

doi: $10.1155 / 2014 / 173939$ [Crossref] 